\title{
Massimo RoSATI, Ritual and Sacred: A Neo- Durkheimian Analysis of Politics, Religion and the Self
}

Farnham, Ashgate, 2009, 163 p.

\section{Enzo Pace}

\section{OpenEdition}

\section{Journals}

Édition électronique

URL : http://journals.openedition.org/assr/22504

DOI : 10.4000/assr.22504

ISSN : $1777-5825$

Éditeur

Éditions de l'EHESS

Édition imprimée

Date de publication : 31 décembre 2010

Pagination : 9-242

ISBN : 9782713223013

ISSN : 0335-5985

\section{Référence électronique}

Enzo Pace, "Massimo rosatı, Ritual and Sacred: A Neo-Durkheimian Analysis of Politics, Religion and the Self », Archives de sciences sociales des religions [En ligne], 152 | octobre-décembre 2010, document 152-102, mis en ligne le 12 mai 2011, consulté le 21 septembre 2020. URL : http:// journals.openedition.org/assr/22504; DOI : https://doi.org/10.4000/assr.22504

Ce document a été généré automatiquement le 21 septembre 2020.

(C) Archives de sciences sociales des religions 


\section{Massimo ROSATI, Ritual and Sacred: A Neo-Durkheimian Analysis of Politics, Religion and the Self}

Farnham, Ashgate, 2009, 163 p.

Enzo Pace

\section{RÉFÉRENCE}

Massimo RosatI, Ritual and Sacred: A Neo-Durkheimian Analysis of Politics, Religion and the Self, Farnham, Ashgate, 2009, 163 p.

1 Le livre de Massimo Rosati, professeur de sociologie à l'Université Tor Vergata de Rome, est publié dans la collection de l'éditeur Ashgate, une collection qui, très significativement, s'intitule «Rethinking Classical Sociology». À l'hérédité et à l'actualité de la pensée de Durkheim, trois textes ont déjà été consacrés si on comprend ce dernier de M.Rosati: le volume de Jonathan Fish (Defending the Durkheimian Tradition) et celui d'Edward Tiryakian (For Durkheim. Essays in Historical and Cultural Sociology). Le fort intérêt du milieu anglo-saxon pour l'œuvre du sociologue français est chose bien connue comme en fait d'ailleurs témoignage la création, déjà en 1991 par Bill Pickering, l'un des plus importants spécialistes de Durkheim, du British Centre for Durkheimian Studies. Rappelons aussi que M.Rosati a également soigné une nouvelle édition critique en italien des Formes, parcourant de nouvelles pistes d'interprétation sur le rapport entre sacré et ritualité.

2 Ce volume se subdivise en six chapitres à travers lesquels l'argumentation s'articule en une séquence logique et sociologique bien précise qui peut être synthétisée comme suit: les Formes constituent le texte fondamental pour comprendre la modernité occidentale, au-delà même des intentions du sociologue français.

3 La leçon durkheimienne, en effet, et en particulier le lien entre rituel et sacré, constitue un paradigme encore valable aujourd'hui pour comprendre pourquoi le rituel et le 
sacré ne sont pas uniquement des éléments de la sphère religieuse mais la grammaire générative du social: des dimensions de la vie quotidienne aux hautes sphères de l'autorité politique et du pouvoir économique. À partir de Durkheim, M. Rosati reconstruit les origines religieuses de l'idée moderne de la conscience introspective du soi individuel capable d'autonomie et d'autoréflexion, parcourant ainsi la piste ouverte par d'autres et, en particulier, celle qui fut explorée par Adam Seligman. Cette analyse est utile à l'auteur pour affirmer sa thèse fondamentale: les contradictions de la modernité ont commencé à se manifester lorsque les sociétés qui, des idéaux de la modernité, non seulement se sont inspirées mais se sont aussi sociétairement parées et organisées (en suivant pour ainsi dire le principe: moins de liens sociaux, plus d'autonomie individuelle), ont peu à peu expulsé le rituel et le sacré de l'horizon de sens des individus.

C'est de là que part M.Rosati pour montrer au contraire combien le couple conceptuel à peine évoqué est à l'origine d'une irréductible infrastructure du social lui-même qui ne peut être éliminée. Même au sein d'une société sécularisée - où les pratiques socioreligieuses deviennent les comportements d'une minorité - la centralité du rituel et du sacré est fondamentale aussi bien pour la construction de l'identité individuelle que pour le maintien des liens sociaux (depuis la chaîne de la mémoire collective jusqu'aux cadres des valeurs qui permettent aux individus d'interagir et de s'entendre éthiquement sur le sens du vivre ensemble). L'idée de M.Rosati est, en d'autres termes, que sans la ritualisation et la conséquente sacralisation d'actions fondamentales de la vie quotidienne et sociale, ne soit plus donnée à l'individu la possibilité de cultiver l'esprit moral qui donne un sens à l'action sociale en tant que telle. Les deux exemples qu'il cite pour expliquer le sens de sa pensée, quand il parle de self-cultivation et d' individual as ceremonial being, sont tirés respectivement de la tradition rabbinique (et ce n'est pas un hasard après avoir porté son regard sur la matrice juive de la pensée durkheimienne) et du confucianisme (qui continue à être bien présent, peut-être de manière latente, dans le fonctionnement d'une méga-société comme l'est la société chinoise).

5 Une fois exposé le cadre analytique au sein duquel l'auteur entend se mouvoir, en remontant à Durkheim pour ensuite le dépasser, M. Rosati se lance dans une tâche difficile: montrer la valeur heuristique de la relation rituel-sacré-société afin de comprendre, d'une part, la crise de légitimation de la politique et, de l'autre, les limites et les contradictions des modèles politiques qui ont prétendu et qui prétendent encore laisser les religions hors de la porte de la sphère publique.

6 La ligne qu'il choisit est à la fois théorique et empirique, en passant de la mise au point de concepts appliqués à chaque fois au domaine religieux et politique, à l'analyse d'études de cas comme le rapport controversé entre islam et politique ou les difficultés que le modèle de la laïcité rencontre quand il se mesure avec le pluralisme religieux inédit que l'Europe dans son ensemble est en train de vivre. Entre le fondamentalisme antimoderne et le libéralisme moderne, M. Rosati semble indiquer une troisième voie: repartir des différentes traditions religieuses pour une entente publique sur des valeurs qui puissent garantir la paix sociale et éviter la reprise de guerres de religion.

7 Sur ce point, je dois dire que nous nous trouvons en pleine mer. Sommes-nous bien certains que l'hérédité de la pensée durkheimienne nous aide à comprendre le fonctionnement d'une société fortement différenciée d'un point de vue culturel et religieux et où les diverses communautés tendent, chacune pour soi, de suivre leur 
propre règle, l'unique bonne et juste, sans reconnaître aux autres le même droit? Ce n'est qu'en séparant le bon du juste, c'est-à-dire en respectant le droit à la différence et le principe de reconnaissance et de légitimation des normes sociales, qui doivent être partagés par tous au-delà des différences, que l'on peut imaginer garantir la cohésion sociale. Il n'est plus possible de se limiter alors à Durkheim pour comprendre la postmodernité: la société qui s'adore elle-même, en réalité, adore aujour-d'hui des divinités différentes (religieuses et séculières); de même que la ritualité s'est décomposée en de si nombreux microrituels (la consommation, les non-lieux du temps libre mais aussi les grands malls de l'esprit où l'on adore un Dieu XXL, pour reprendre une belle formule de Sébastien Fath qui s'est penché sur les méga-églises là où le nombre des présents est si élevé que ce n'est pas tant le lien social qui compte mais plutôt la satisfaction narcissique de l'individu qui accède à une performance liturgique personnelle) que les contours des rites sociaux de passage en deviennent vagues et incertains. Un polythéisme des valeurs qui rappelle Weber et auquel la pensée néodurkheimienne ne peut se soustraire. 